Colloque C2, suppl. au Journal de Physique II, Vol 1, septembre 1991

\title{
ANGULAR DISTRIBUTION OF DESORBED MOLECULES
}

\author{
X.W. LI, B.Z. GUO, S.H. LIN* and H. MA* \\ Department of Electronics, Hebei University, Baoding 071002 , \\ $P . R$. China \\ *Department of Chemistry, Arizona state University, Tempe, $A Z$ \\ 85287-1604, U.S.A.
}

\begin{abstract}
A macroscopic theory for treating the angular distibution of desorptive products due to thermal or photo-assisted desorption is reported. The angular distribution has been attributed to either the rotational relaxation of adsorbed molecules or the angular dependence of the rate constant or both. By using the theory of angular momentum, explicit expressions of the desorption yields for the cases of constant temperature or heating with a constant rate were obtained. Based on the theory, some experimental results such as the desorption of $\mathrm{CO}_{2}$ from $\mathrm{Pt}(111)$ surface have been explained and the effect of crystal orientation of the substrates was discussed as well.
\end{abstract}

\section{I . INTRODUCTION.}

In the CVD of thin films, the desorption of adsorbed species from the surface of substrate is an important elemental process, which may even control the deposition rate,for instance, in the desorption of hydrogen atoms for silicon deposition from silane. /1/ However, the studies of desorption mechanism have been not so intensive as that of other elemental processes such as gaseous transmission and crystal growth. Generally, the desorption occured in CVD belongs to thermal activated process and can be described phenomenologically with Arrhenius formula. Since the early work on thermal desorption $/ 2 /$ there have been a number of experimental studies of the angular distribution of desorbing molecules $/ 3-9 /$. In his review Comsa et al. pointed out that, the cosine dependence of desorption flux on the polar angle is an elementary property of molecule emission process, but several actual angular dependence of desorption deviated from cosine distribution and were traditionally described by $\cos ^{n} \theta$ without any physical background. In particular, the system of $\mathrm{H}_{2}$ $/ \mathrm{Ni}(111)$ has attracted considerable attention. When the $\mathrm{Ni}(111)$ surface has been 
saturated with adsorbed $H$ at $T=140 \mathrm{~K}$ and is then heated at $2 \mathrm{~K} / \mathrm{sec}$, two thermal desorption features $\beta_{1}(T=290 \mathrm{~K})$ and $\beta_{2}(T=370 \mathrm{~K})$ are sequentially desorbed. The two states are found to have distinctly different angular distributions. $\beta_{2}-\mathrm{H}_{2}$ desorption is strongly focused along the surface normal (that can be described by $(\cos \theta)^{9 / 2}$ ), while $\beta_{1}-\mathrm{H}_{1}$ desorbs diffusely (that $\operatorname{can}$ be described by $\cos \theta$ ). A similar example is the angular distribution of $\mathrm{CO}_{2}$ desorbing as the product of the oxidation of $\mathrm{CO}$ incident on a Pt(111) surface precovered with oxygen /10/. Segner et al. succeeded in fitting all their $\mathrm{CO}_{2}$ desorption data by using a expression of the type

$$
I_{\mathrm{Co}_{2}}(\theta)=\mathrm{a} \cos \theta+(1-a) \cos ^{7} \theta
$$

It is obvious that the experimental results were even not fitted by a simple $\cos ^{n} \theta$ and required a essentially physical explaination.

In this paper,we shall propose a theoretical approach which based on consideration of the physical states of adsorbed molecules on a surface to explain the angular distribution of desorbing molecules.

\section{II . ANGULAR DISTRIBUTION OF THERMAL DESORPTION}

It has been demonstrated from experiments that the desorbed molecules carry with themself a certain amount of rotational energy due to vibration-rotation relaxation. $/ 11,12 /$ In other words, when a surface at equilibrium was heated or exposed to laser the molecules adsorbed on the surtace would undergo transitions between their rotational states and a process for redistributing their energy, while the adsorbed molecules may correspondingly change their orientation. Acoording to Dedye $/ 13 /$, the orientational relaxation of the adsorbed molecules can be described by rotational diffusion and we attribute the difference of the desorption mechanisms to an orientation dependence of the desorption rate constant. Thus for the first-order reaction case, we have the diffusion equation as

$$
\begin{gathered}
\frac{\partial A}{\partial t}=D \nabla^{2} A-K(\theta) A \\
\nabla^{2}=\frac{1}{\sin \theta \partial \theta}\left(\sin \theta \frac{\partial}{\partial \theta}\right)+\frac{1}{\sin ^{2} \theta} \partial^{2}
\end{gathered}
$$

Where $D$ and $K(\theta)$ are the diffusion coefficient of rotation (i. e. the rate of the orientational relaxation) and the desorption rate constant, respectively; $A$ is the density of the adsorbed molecules which is generally a function of time and the orientational angle relative to the surface normal. 
Whithout the presence of an external field, the system is independent of $\phi$. In this case to solve Eq. (2), we expand $A(\theta, t)$ with normalized Legendre polynomial $Y_{L}$ ( $\theta)$ as follows

$$
A(\theta, t)=\underset{L}{A_{L}}(t) Y_{L}(\theta)
$$

Substituting Eq. (4) into eq. (2) yields

$$
\frac{\partial A_{L}}{\partial t}=-\mathbf{L}(L+1) D A_{L}-\Sigma_{L^{\prime}} A_{L^{\prime}}\left\langle Y_{L}|K(\theta)| Y_{L^{\prime}}\right\rangle
$$

where

$$
\left\langle Y_{L}|K(\theta)| Y_{L^{\prime}}\right\rangle=\int_{0}^{n} \sin \theta d \theta Y_{L}^{*}(\theta) K(\theta) Y_{L^{\prime}}(\theta)
$$

First, we consider the case of $\mathrm{K}(\theta)=\mathrm{K}$, i. e the desorption rate is independent with the orientational angle. Then the integration in Eq. (5) can be carried out and we have

$$
A_{L}(t)=A_{L}(0) \exp \left\{-\int_{0}^{t} d t^{\prime}[K+L(L+1) D]\right\}
$$

where $K$ and $D$ are not apparent functions of time, but depend on temperature. In the condition of constant temperature,Eq. (7) becomes

$$
A_{L}(t)=\frac{A_{L}(0)}{K+L(L+1) D}\left\{1-e^{-[K+L(L+1) D] t}\right\}
$$

thereby the desorption yield

$$
P=K A(\theta, t)=\sum_{1} \frac{A_{L}(0) Y_{L}(\theta)}{1+L(L+1) D / K}\left\{1-e^{-[K+L(L+1) D] t}\right\}
$$

and the accumulative yield obtained by long time observed is

$$
I=\lim _{\mathrm{T} \rightarrow \infty} \int_{0}^{\mathrm{T}} \mathrm{P}(\mathrm{t}) \mathrm{dt}
$$

where the integration start from the moment when the equilibrium condition of the surface changes.

When the temperature changes with the rate $\gamma_{3}=\frac{d T}{d t}$, Eq. (9) will take the following form

$$
\mathbf{P}=\mathrm{K} \sum_{\mathrm{L}} \mathrm{A}_{\mathbf{L}}(0) \mathrm{Y}_{\mathrm{L}}(\theta) \exp \left\{-\frac{1}{\gamma} \int_{0}^{T} \mathrm{~d} \mathrm{~T}^{\prime}\left[\mathrm{K}\left(\mathrm{T}^{\prime}\right)+\mathrm{L}(\mathrm{L}+1) \mathrm{D}\left(\mathrm{T}^{\prime}\right)\right]\right\}
$$

It can be seen from Eqs. (9) and (11) that this is a competing process in which the rotational relaxation and the desorptive reaction participate. In the expanding expression, the coefficients of $\mathrm{Y}^{\prime}{ }_{\mathrm{L}} \mathrm{s}$ with larger value of $\mathrm{L}$ decrease faster with time. This means the angular distribution of desorptive products approaches $Y_{0}$, the Lambert 
emisstion, due to effect of the relaxation toward the atate of $L=0$. However, if the diffusion coefficent of rotation is small, the angular distribution of desorption products is determined by the initial distribution of adsorbed molecules.

When the desorption rate is angle-dependent, generally, $\mathrm{K}(\theta)$ can be expanded as

$$
K(\theta)=\sum K_{m} Y_{m}(\theta)
$$

Thus the solution for Eq. (2) is

$$
\frac{\mathrm{dA}_{\mathrm{L}}}{\mathrm{dt}}=-\mathbf{L}(\mathrm{L}+1) \mathrm{DA}_{\mathrm{L}}-\sum_{\mathrm{L}^{\prime}} \sum_{\mathrm{m}} \mathrm{K}_{\mathrm{m}} \mathrm{A}_{\mathrm{L}^{\prime}}\left\langle\mathrm{Y}_{\mathrm{L}}\left|\mathrm{Y}_{\mathrm{m}}\right| \mathrm{Y}_{\mathrm{L}^{\prime}}\right\rangle
$$

It has been well known from Wigner-Eckart theorem $/ 14 /$ that

$$
\left\langle Y_{L}\left|Y_{m}\right| Y_{L^{\prime}}\right\rangle=\left[\frac{\left(2 L^{\prime}+1\right)(2 m+1)}{4 \pi(2 L+1)}\right]^{\frac{1}{2}} C^{2}\left(L^{\prime} m L ; 000\right)
$$

where $\mathrm{C}\left(\mathrm{L}^{\prime} \mathrm{mL} ; 000\right)$ denotes Clebsch-Gordan coefficient.

\section{III . DISCUSSION}

We first disuss an extreme case where the rotational relaxation is much faster than the reaction (or desorption), . Then the molecule will relax to the $L=0$ state (i. e. isotropic) before the reaction takes place. In the case, we have

$$
\dot{A}(\theta, t)=A_{0} \exp (-K(\theta) t)
$$

That is, in the case the angular distribution of thermal desorption is mainly due to the angular dependence of the reaction rate constant.

For colparison with experimental results, we have to cimplify Eq. (13). It should be pionted out that there exist some selection rules corresponding to the reactive types and they can be used to solve Eq. (13). For example,if $K(\theta)$ contains only the term of $Y_{1}(\theta)$, $i, e$, for the case of desorption resulted from absorbing single photon,Eq. (13) can be simlpified into

$$
\frac{d A_{L}}{d t}=-L(L+1) D A_{L}-\sum_{L^{\prime}} L^{\prime}\left(L^{\prime}+1\right) K_{1} A_{L^{\prime}} C\left(L^{\prime} 1 L ; 000\right)
$$

here it is remained only two terms of Clebsch-Gordan coefficients, i. e. the terms of $\mathbf{L}^{\prime}=\mathbf{L}+1$ and $\mathbf{L}^{\prime}=\mathbf{L}-1$. Actually, Eq. (16) represents a set of couple differential equations which can be solved by using the perturbation method.

Another case allowed to solve Eq. (13) is $A_{L}(O)=A(O) \delta_{L S}$,i.e. if the initial angular distribution of adsorbed molecules has the form of $\mathrm{Y}_{s}(\theta)$, we obtain a expression 
of accumulative yields as

$$
\begin{aligned}
& I=A(0) \sum_{p=-L-m}^{L+m} \sum_{L=s-m^{\prime}}^{s+m^{\prime}} \sum_{m \cdot m^{\prime}} K_{m} K_{m^{\prime}}\left[\frac{(2 s+1)\left(2 m^{\prime}+1\right)}{4 \pi(2 L+1)}\right]^{\frac{1}{2}} \times \\
& \frac{C^{2}\left(s m^{\prime} L ; 000\right) C(L m p ; 000)}{\left[s(s+1) D+K_{m}\right][L(L+1) D]} Y_{p}(\theta),
\end{aligned}
$$

which fives a series with highest power term of $(\cos \theta)^{\mathbf{L}+m}$. Notice that, here $\mathbf{L}$ and $m$ represent orientation features of adsorbed molecules and the desorption reaction, respectively. To our knowledge, there has not been any experimental evidence of the high power dependence of coeffor the desorption reaction. However, a couple of researchers reported a strong dependence of the angular distribution of desorbing molecules on the surface precover, $/ 10 /$ where their very different data showm a commom tendency, the angular distribution of $\mathrm{H}_{2}$ desorption from a $\mathrm{Cu}$-covered Ni(110) surface becomes much sharper with increasing the srufcace coverage $\theta$ . Otherwise, for a different crystal orientation of substrates, say $\mathrm{Ni}(111)$, the dependence is not so sensitive. These suggest to consider a shaden effect between closed molecules and the important role of crystal orientation. Particularly, for $m=m^{\prime}=1$ and $s=5$, Eq. (17) can be simplified and becomes $I=I_{0}\left(Y_{7}-c_{1}, Y_{5}+c_{2}, Y_{3}\right)$. Selecting suitable parameters $\mathrm{K}$ and $\mathrm{D}$ can yield Eq. (1) and fit the experimental data well.

Up to mow we discussed only for the first-order reaction. However, the theoretical approach could be used to any types of desorption processes. Here we consider the case of zeroth order reactions

$$
\frac{\partial A_{L}}{\partial t}=D \nabla^{2} A-K(\theta)
$$

Using Eq. (4), we obtain

$$
\frac{\mathrm{dA}_{\mathrm{L}}}{\mathrm{dt}}=-\mathrm{DL}(\mathrm{L}+1) \mathrm{A}_{\mathbf{L}}-\mathrm{K}_{\mathbf{L}}
$$

It is easy to integrate Eq. (19) and for the general case we obtain

$$
\begin{aligned}
& A(\theta, t)=\left[A_{0}(0)-\int_{0}^{t} d t^{\prime} K_{0}\left(t^{t}\right)\right] Y_{0}(\theta)+\sum_{L=1} Y_{L}(\theta)\left[A_{L}(0)\right. \\
& \left.\int_{0}^{t} d t^{\prime} K_{L}\left(t^{\prime}\right) \exp \left\{L(L+1) \int_{0}^{t} d t^{\prime} D\right\}\right] \exp \left[-L(L+1) \int_{0}^{t} d t^{\prime} D(t)\right]
\end{aligned}
$$

Therefore, the desorption yields could be computed numerically as long as the initial angular distribution and the desorption rate constant as a function of time(through temperature) were known.

As a conclusion we mention that the theoretical approach could deal with thermal or optical desorption process and explain more complex angular distribution of desorbed 
molecules with the physical background. Especially, the dynamic results of the theory are just suitable for CVD process, where during deposition the desorption always occures. As well known, desorption is a inverse process of deposition, therefore, from the desorption measurement ones can obtain the knowledge about bonded atates and orientation of the molecules in deposited films.

\section{REFERENCES}

/1/ Li Xing-Wen and Wang Ying-Min, J. Hebei Univ, , 3(1983) 1

/2/ W. Van Willigan, Phys. Lett. , A28 (1968) 80

$/ 3 /$ G. Comsa and R. David,Surface Sci. Reports,5(4) (1985) 145

/4/ H. P. Steinruck, M. Luger, A. Winkler and K. D. Rendulic, Phys. Rev. ,B32 (1985) 5032

$/ 5 /$ H. J. Robota, M. C. Lin and G. Ertl,Surf.Sci. ,155(1985) 101

/6/ J. N. Russell, Jr. ,I. Chorkendorff, A. M. Lanzillotto,M. D. Alvey and J. T. Yates, Jr. ,J. Chem. Phys. , 85(1986)6186

$/ 7 /$ D. O. Hayward and A. D. Taylor, Chem. Phys. Lett. , 124(1986) 264

$/ 8 /$ N. S. Gluck. Z. Ying, C. E. Bartosch and W. Ho, J. Chem. Phys. ,86 (1987) 4987

/9/ T. Matsushima, J. Phys, Chem. ,91(1987) 6192

$/ 10 /$ J.Segner,C. T. Campbell, G. Doyen and G. Ertl, Surf. sci. , 138 (1984) 505

H. P. Steinruck, A. Winkler and K. D. Rendulic, J. Phys. C, 17 (1984) L311

/11/ R.P. Thorman and S. L. Bermasek, J. Chem. Phys. , 74 (1981) 6498

$/ 12 /$ R. R. Cavanagh and D. S. King, J. Vacuum Sci. Technol. ,A1(1983) 1267

$/ 13 /$ P. Debye, "Polar Molecules" chapter 5, Dover, (1928)

/14/M.E. Rose. "Elementary Theory of Angular Momentum", John Wihey \& Sons, Inc. , (1957) 